Article

\title{
Analytical Investigations on Carrier Phase Recovery in Dispersion-Unmanaged $n$-PSK Coherent Optical Communication Systems
}

\author{
Tianhua $\mathrm{Xu}^{1,2, *}$, Gunnar Jacobsen ${ }^{3,4}$, Sergei Popov ${ }^{4}$, Jie $\mathrm{Li}^{3}$, Tiegen Liu ${ }^{2}$, Yimo Zhang ${ }^{2}$ \\ and Polina Bayvel ${ }^{1}$ \\ 1 Optical Networks Group, Department of Electronic \& Electrical Engineering, University College London, \\ London WC1E 7JE, UK; p.bayvel@ucl.ac.uk \\ 2 Department of Optical Engineering, Tianjin University, Tianjin 300072, China; tgliu@tju.edu.cn (T.L.); \\ ymzhang@tju.edu.cn (Y.Z.) \\ 3 Acreo Swedish ICT AB, Stockholm SE-16425, Sweden; gunnar.jacobsen@acreo.se (G.J.); Jie.Li@acreo.se (J.L.) \\ 4 Royal Institute of Technology, Stockholm SE-16440, Sweden; sergeip@kth.se \\ * Correspondence: tianhua.xu@ucl.ac.uk or xutianhua@tju.edu.cn; Tel.: +44-770-966-2611
}

Received: 22 August 2016; Accepted: 21 September 2016; Published: 24 September 2016

\begin{abstract}
Using coherent optical detection and digital signal processing, laser phase noise and equalization enhanced phase noise can be effectively mitigated using the feed-forward and feed-back carrier phase recovery approaches. In this paper, theoretical analyses of feed-back and feed-forward carrier phase recovery methods have been carried out in the long-haul high-speed $n$-level phase shift keying (n-PSK) optical fiber communication systems, involving a one-tap normalized least-mean-square (LMS) algorithm, a block-wise average algorithm, and a Viterbi-Viterbi algorithm. The analytical expressions for evaluating the estimated carrier phase and for predicting the bit-error-rate (BER) performance (such as the BER floors) have been presented and discussed in the $n$-PSK coherent optical transmission systems by considering both the laser phase noise and the equalization enhanced phase noise. The results indicate that the Viterbi-Viterbi carrier phase recovery algorithm outperforms the one-tap normalized LMS and the block-wise average algorithms for small phase noise variance (or effective phase noise variance), while the one-tap normalized LMS algorithm shows a better performance than the other two algorithms for large phase noise variance (or effective phase noise variance). In addition, the one-tap normalized LMS algorithm is more sensitive to the level of modulation formats.
\end{abstract}

Keywords: coherent optical detection; optical fiber communication; carrier phase recovery; feed-back and feed-forward; laser phase noise; equalization enhanced phase noise; $n$-level phase shift keying

\section{Introduction}

Since the first generation of optical fiber communication systems was deployed over 30 years ago, the achievable data rates carried by a single optical fiber have been raised over 10,000 times, and the data network traffic has also been increased by over a factor of 100 [1,2]. To date, more than $90 \%$ of digital data is transmitted over optical fibers, to constitute the greater part of national and international telecommunication infrastructures. The effective information capacity of these networks has been widely increased over the past three decades with the introduction and development of wavelength division multiplexing (WDM), higher-level modulation formats, digital signal processing (DSP), advanced optical fibers and amplification technologies [3,4]. These developments promoted the revolution of communication systems and the growth of the Internet, towards the direction of higher-speed and longer-distance transmissions [2,3]. The performance of long-haul high-speed optical fiber communication systems can be significantly degraded by the impairments in the transmission 
channels and laser sources, such as chromatic dispersion (CD), polarization mode dispersion (PMD), laser phase noise (PN) and fiber nonlinearities (FNLs) [4-8]. Using coherent optical detection and digital signal processing, the powerful equalization and effective mitigation of the communication system impairments can be implemented in the electrical domain [9-18], which has become one of the most promising techniques for the next-generation optical fiber communication networks to achieve a performance very close to the Shannon capacity limit $[19,20]$, with an entire capture of the amplitude and phase of the optical signals. Using high-level modulation formats such as the $n$-level phase shift keying (n-PSK) and the $n$-level quadrature amplitude modulation ( $n$-QAM), the performance of optical fiber transmission systems will be degraded seriously by the phase noise from the transmitter (Tx) lasers and the local oscillator (LO) lasers [21,22]. To compensate the phase noise from the laser sources, some feed-forward and feed-back carrier phase recovery (CPR) approaches have been proposed to estimate and remove the phase of optical carriers [23-32]. Among these carrier phase estimation (CPE) methods, the one-tap normalized least-mean-square (LMS) algorithm, the block-wise average (BWA) algorithm, and the Viterbi-Viterbi (VV) algorithm have been validated for mitigating the laser phase noise effectively, and are also regarded as the most promising DSP algorithms in the real-time implementation of the high-speed coherent optical fiber transmission systems [27-32]. Thus it will be of importance and interest to investigate the performance of these three carrier phase recovery algorithms in long-haul high-speed optical communication systems.

In electronic dispersion compensation (EDC) based coherent optical fiber communication systems, an effect of equalization enhanced phase noise (EEPN) will be generated due to the interactions between the electronic dispersion equalization module and the laser phase noise (in the post-EDC case the EEPN comes from the LO laser) [33-38]. The performance of long-haul optical fiber communication systems will be degraded seriously due to the equalization enhanced phase noise, with the increment of fiber dispersion, laser linewidths, modulation levels, symbol rates and system bandwidths [33-36]. The impacts of EEPN have been investigated in the single-channel, the WDM multi-channel, the orthogonal frequency division multiplexing (OFDM), the dispersion pre-distorted, and the multi-mode optical fiber transmission systems [39-46]. In addition, some investigations have been carried out to study the influence of EEPN in the carrier phase recovery in long-haul high-speed optical communication systems [47-50]. Considering the equalization enhanced phase noise, the traditional analyses of the carrier phase recovery approaches are not suitable any longer for the design and the optimization of long-haul high-speed optical fiber networks. Therefore, it will also be interesting and useful to investigate the performance of the one-tap normalized LMS, the block-wise average, and the Viterbi-Viterbi carrier phase recovery algorithms, when the influence of equalization enhanced phase noise is taken into account.

In previous reports, the analytical derivations and numerical studies for the one-tap normalized LMS, block-wise average, and Viterbi-Viterbi carrier phase recovery methods have been carried out based on the quadrature phase shift keying (QPSK) coherent optical transmission system [26,37,51]. However, with the development of the optical fiber networks and the increment of transmission data capacity, the QPSK modulation format can no longer satisfy the demand for high-speed optical fiber communication systems. Therefore, the analyses on the carrier phase recovery approaches should also be updated accordingly for the optical fiber transmission systems using higher-level modulation formats, such as the $n$-PSK communication systems.

In this paper, built on the previous work in $[26,37,51]$, the theoretical assessments of the carrier phase recovery using the one-tap normalized LMS, the block-wise average, and the Viterbi-Viterbi algorithms are extended and analyzed in detail for the long-haul high speed $n$-PSK coherent optical fiber communication systems, considering both the intrinsic laser phase noise and the equalization enhanced phase noise. The analytical expressions for the estimated carrier phase in the one-tap normalized LMS, the block-wise average, and the Viterbi-Viterbi algorithms has been derived, and the bit-error-rate (BER) performance such as the BER floors in these three carrier phase recovery approaches has been predicted for the $n$-PSK coherent optical transmission systems. Our results indicate that 
the Viterbi-Viterbi carrier phase recovery algorithm outperforms the one-tap normalized LMS and the block-wise average algorithms for small phase noise variance (or effective phase noise variance), while the one-tap normalized LMS carrier phase recovery algorithm shows a better performance than the other two algorithms for large phase noise variance (or effective phase noise variance). It is also found that the one-tap normalized LMS algorithm is more sensitive to the level of the modulation formats than the other two algorithms.

\section{Laser Phase Noise and Equalization Enhanced Phase Noise}

As shown in Figure 1, the origin of equalization enhanced phase noise in the coherent optical communication systems using electronic dispersion compensation and carrier phase recovery is schematically illustrated. In such systems, the transmitter laser phase noise goes through both the transmission fiber and the EDC module, and therefore the net dispersion experienced by the transmitter laser phase noise is close to zero. However, the LO laser phase noise only goes through the EDC module, where the transfer function is heavily dispersed in the transmission system without using any optical dispersion compensation (ODC) techniques. As a result, the LO laser phase noise will interplay with the dispersion equalization module, and will significantly degrade the performance of the long-haul high-speed coherent optical fiber communication systems, with the increment of fiber dispersion, laser linewidths, modulation formats, and symbol rates [33,34,36].

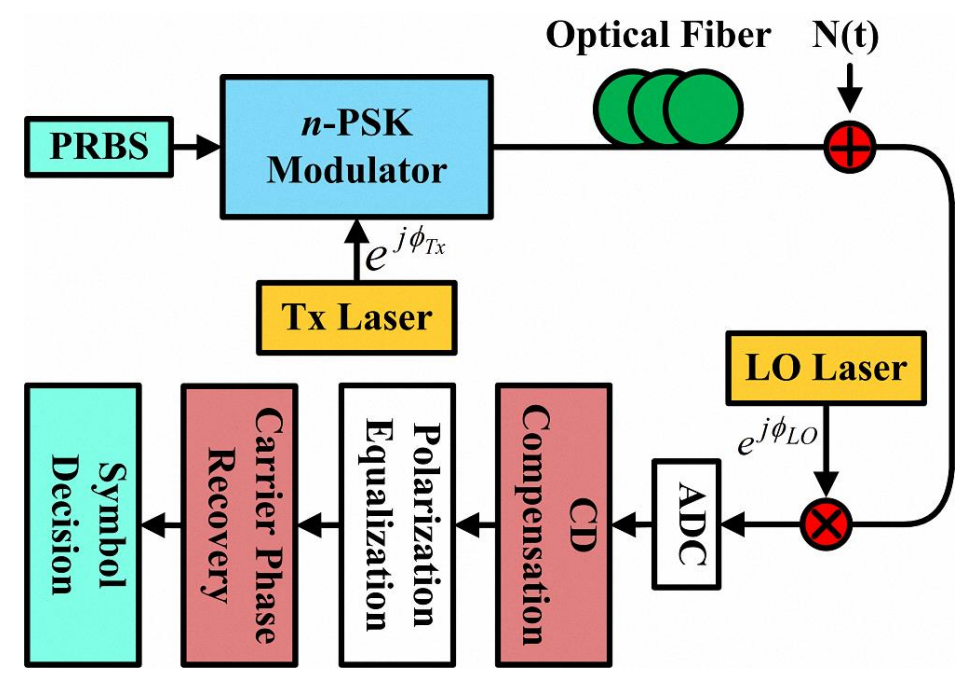

Figure 1. Principle of equalization enhanced phase noise in electronic dispersion compensation based $n$-PSK coherent optical transmission system. PRBS: pseudo random bit sequence, $\mathrm{N}(\mathrm{t})$ : additive white Gaussian noise (AWGN), e.g., amplified spontaneous emission (ASE) noise from optical amplifiers, ADC: analog-to-digital convertor.

In coherent optical communication systems, the variance of the phase noise coming from the transmitter laser and the LO laser follows a Lorentzian distribution and can be described using the following equation $[4,5]$ :

$$
\sigma_{\text {Laser }}^{2}=2 \pi\left(\Delta f_{T x}+\Delta f_{L O}\right) \cdot T_{S}
$$

where $\Delta f_{T x}$ and $\Delta f_{L O}$ are the 3-dB linewidths (assuming the Lorentzian distribution) of the transmitter laser and the LO laser, respectively, and $T_{S}$ is the symbol period of the coherent transmission system. It can be found that the variance of the laser phase noise decreases with the increment of the signal symbol rate $R_{S}=1 / T_{S}$.

Considering the interplay between the electronic dispersion compensation module and the LO laser phase noise, the noise variance of the equalization enhanced phase noise in the long-haul high-speed optical fiber communication systems can be expressed as follows $[33,37,40]$ : 


$$
\sigma_{E E P N}^{2}=\pi \lambda^{2} D \cdot L \cdot \Delta f_{L O} / 2 c T_{S},
$$

where $f_{L O}$ is the central frequency of the LO laser, which is equal to the central frequency of the transmitter laser $f_{T x}$ in the homodyne optical communication systems, $D$ is the CD coefficient of the transmission fiber, $L$ is the length of the transmission fiber, $R_{S}$ is the signal symbol rate of the communication system, and $\lambda=c / f_{T x}=c / f_{L O}$ is the central wavelength of the optical carrier wave.

When the equalization enhanced phase noise is taken into account in the carrier phase recovery, the total noise variance (or effective phase noise variance) in the long-haul high-speed $n$-PSK optical fiber transmission systems can be calculated and described using the following expression [37,40]:

$$
\sigma_{T}^{2} \approx \sigma_{\text {Laser }}^{2}+\sigma_{E E P N}^{2}=2 \pi T_{S}\left(\Delta f_{T x}+\Delta f_{L O}\right)+\pi \lambda^{2} D \cdot L \cdot \Delta f_{L O} / 2 c T_{S}
$$

The equalization enhanced phase noise differs from the laser phase noise, and the noise variance in Equation (2) has two-thirds contribution in the phase noise and one-third contribution in the amplitude noise $[33,37,40]$. Therefore, Equation (3) is only valid for $n$-PSK communication systems, and the performance of $n$-QAM transmission systems has to be assessed based on the evaluation of error vector magnitudes [52].

Corresponding to the definition of the laser phase noise from the transmitter and the LO lasers, an effective linewidth $\Delta f_{E f f}$ can be employed to describe the total phase noise variance in the EDC-based $n$-PSK coherent optical communication systems and it can be expressed as follows:

$$
\Delta f_{E f f} \approx\left(\sigma_{\text {Laser }}^{2}+\sigma_{E E P N}^{2}\right) / 2 \pi T_{S} .
$$

When the impact from laser phase noise and the influence from EEPN give an equal contribution in the $n$-PSK optical fiber communication systems, namely $\sigma_{\text {Laser }}^{2}=\sigma_{E E P N}^{2}$, we will have the transmission distance of $L_{0}=8 c T_{S}^{2} / \lambda^{2} D$.

Take the 32-Gbaud $n$-PSK coherent optical fiber transmission system as an example, and we assume that the fiber CD coefficient is $17 \mathrm{ps} / \mathrm{nm} / \mathrm{km}$ and the central wavelengths of the transmitter and the LO lasers are both $1550 \mathrm{~nm}$. In this case, we have $L_{0}=60.69 \mathrm{~km}$. It means that at this transmission distance, the laser phase noise and the EEPN will have the same impact on the degradation of the performance of the 32-Gbaud $n$-PSK optical transmission systems.

\section{Analysis of Carrier Phase Recovery Approaches}

\subsection{One-Tap Normalized Least-Mean-Square (LMS) Carrier Phase Recovery}

As a feed-back carrier phase recovery approach $[27,28]$, which is schematically shown in Figure 2, the transfer function of the one-tap normalized LMS algorithm in the $n$-PSK coherent optical communication systems can be expressed using the following equations:

$$
\begin{gathered}
y(k)=w_{N L M S}(k) x(k), \\
w_{N L M S}(k+1)=w_{N L M S}(k)+\mu e(k) x^{*}(k) /|x(k)|^{2}, \\
e(k)=d(k)-y(k),
\end{gathered}
$$

where $x(k)$ is the complex input symbol, $k$ is the index of the symbol, $y(k)$ is the complex output symbol, $w_{N L M S}(k)$ is the tap weight of the one-tap normalized LMS equalizer, $d(k)$ is the desired output symbol after the carrier phase recovery, $e(k)$ is the estimation error between the output symbol and the desired output symbol, and $\mu$ is the step size of the one-tap normalized LMS algorithm.

It has been verified that the optimized one-tap normalized LMS carrier phase recovery in the QPSK optical transmission systems behaves similarly to the ideal differential carrier phase recovery [24,37], 
and the BER floor of the one-tap normalized LMS carrier phase recovery in the QPSK transmission systems can be approximately described as follows [37]:

$$
B E R_{\text {floor }}^{N L M S_{-} Q P S K} \approx \frac{1}{2} \operatorname{erfc}\left(\frac{\pi}{4 \sqrt{2} \sigma_{T}}\right) .
$$

Therefore, the BER floor of the one-tap normalized LMS carrier phase recovery for the $n$-PSK optical fiber communication systems can be derived accordingly, and can be expressed using the following equation:

$$
B E R_{\text {floor }}^{N L M S} \approx \frac{1}{\log _{2} n} \operatorname{erfc}\left(\frac{\pi}{n \sqrt{2} \sigma_{T}}\right),
$$

where $\sigma_{T}^{2}$ is the total phase noise variance (or effective phase noise variance) in the long-haul high-speed n-PSK optical transmission systems.

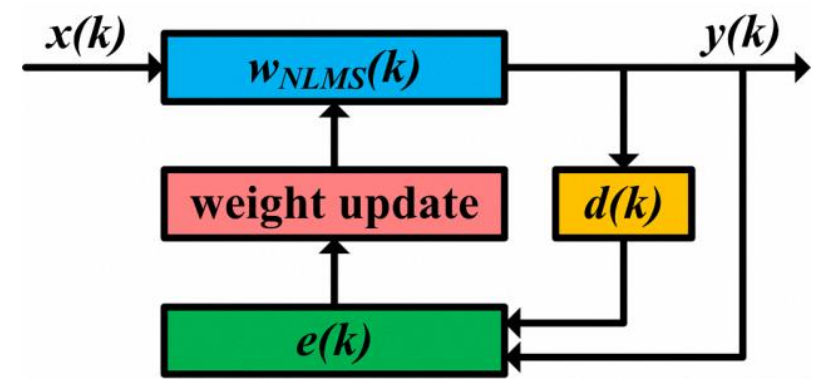

Figure 2. Schematic of one-tap normalized LMS carrier phase recovery algorithm.

\subsection{Block-Wise Average Carrier Phase Recovery}

As an $n$-th power feed-forward carrier phase recovery approach, which is schematically shown in Figure 3, the block-wise average algorithm calculates the $n$-th power of the received symbols to remove the information of the modulated phase in the $n$-PSK coherent transmission systems, and the computed phase ( $n$-th power) data are summed and averaged over a certain block. The averaged phase value is then divided over $n$, and the final result is regarded as the estimated phase for the received symbols within the entire block $[29,30]$. For the $n$-PSK coherent optical communication systems, the estimated carrier phase for each process block using the block-wise average algorithm can be expressed as:

$$
\begin{gathered}
\varphi_{B W A}(k)=\frac{1}{n} \arg \left\{\sum_{p=1+(q-1) \cdot N_{B W A}}^{q \cdot N_{B W A}} x^{n}(p)\right\}, \\
q=\left\lceil k / N_{B W A}\right\rceil,
\end{gathered}
$$

where $k$ is the index of the received symbol, $N_{B W A}$ is the block length in the block-wise average algorithm, and $\lceil x\rceil$ means the closest integer lager than $x$.

The BER floor of the block-wise average carrier phase recovery in the $n$-PSK coherent optical communication systems can be derived using the Taylor series expansion, and can be approximately described using the following equation:

$$
\begin{gathered}
B E R_{\text {floor }}^{B W A} \approx \frac{1}{N_{B W A} \log _{2} n} \cdot \sum_{p=1}^{N_{B W A}} \operatorname{erfc}\left(\frac{\pi}{n \sqrt{2} \sigma_{B W A}(p)}\right), \\
\sigma_{B W A}^{2}(p)=\frac{\sigma_{T}^{2}\left[2(p-1)^{3}+3(p-1)^{2}+2\left(N_{B W A}-p\right)^{3}+3\left(N_{B W A}-p\right)^{2}+N_{B W A}-1\right]}{6 N_{B W A}^{2}},
\end{gathered}
$$


where $\sigma_{T}^{2}$ is the total phase noise variance (or effective phase noise variance) in the long-haul high-speed $n$-PSK optical transmission systems.

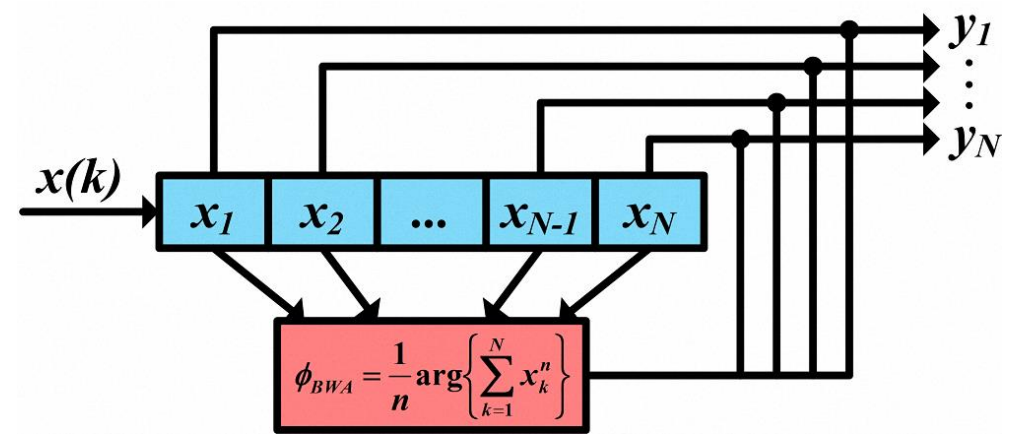

Figure 3. Schematic of block-wise average carrier phase recovery algorithm.

\subsection{Viterbi-Viterbi Carrier Phase Recovery}

As another $n$-th power feed-forward carrier phase recovery approach, which is schematically shown in Figure 4, the Viterbi-Viterbi algorithm also calculates the $n$-th power of the received symbols to remove the information of the modulated phase. The computed phase data are also summed and averaged over the processing block (with a certain block length). Compared to the block-wise average algorithm, the Viterbi-Viterbi algorithm just treats the extracted phase as the estimated phase for the central symbol in each processing block [31,32]. The estimated carrier phase in the Viterbi-Viterbi algorithm in the $n$-PSK coherent optical transmission systems can be described using the following equation:

$$
\varphi_{V V}(k)=\frac{1}{n} \arg \left\{\sum_{q=-\left(N_{V V}-1\right) / 2}^{\left(N_{V V}-1\right) / 2} x^{n}(k+q)\right\},
$$

where $N_{V V}$ is the block length of the Viterbi-Viterbi algorithm, and should be an odd value of e.g., 1, 3, $5,7 \ldots$

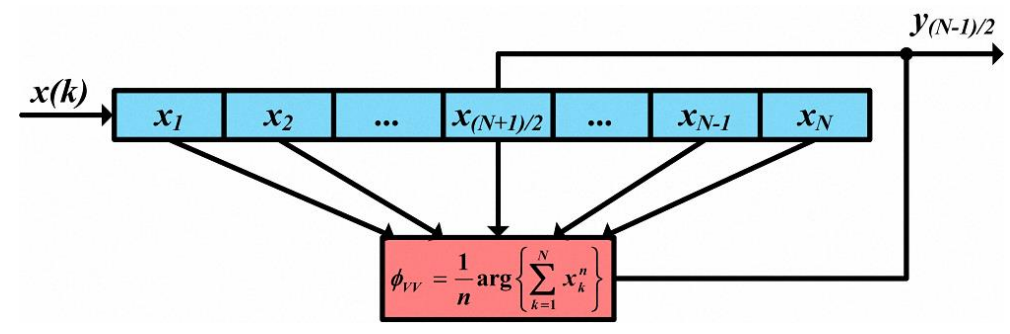

Figure 4. Schematic of Viterbi-Viterbi carrier phase recovery algorithm.

Using the Taylor expansion, the BER floor of the Viterbi-Viterbi carrier phase recovery in the $n$-PSK coherent optical communication systems can be assessed analytically, and can be expressed approximately using the following equation:

$$
B E R_{\text {floor }}^{V V} \approx \frac{1}{\log _{2} n} \operatorname{erfc}\left(\frac{\pi}{n \cdot \sqrt{\frac{N_{V V}^{2}-1}{6 N_{V V}} \cdot \sigma_{T}}}\right),
$$

where $\sigma_{T}^{2}$ is the variance of the total phase noise (or effective phase noise) in the long-haul high-speed $n$-PSK optical fiber transmission systems. 


\section{Results and Discussion}

\subsection{Results}

In this section, the performance of carrier phase recovery in the long-haul high-speed $n$-PSK coherent optical transmission systems using the one-tap normalized LMS, the block-wise average, and the Viterbi-Viterbi algorithms are investigated based on the above theoretical studies, which can be regarded as extensions of the QPSK results in previously reported work [26,37,51]. The comparisons of the three carrier phase recovery approaches have also been carried out in detail. In all these analyses and discussions, the standard single mode fiber (SSMF) has been employed in the $n$-PSK coherent optical transmission systems, where the fiber dispersion is $17 \mathrm{ps} / \mathrm{nm} / \mathrm{km}$, the central wavelengths of both the transmitter laser and the LO laser are $1550 \mathrm{~nm}$, and the signal symbol rate is 32-Gbaud. The fiber attenuation, the PMD, and the nonlinear effects are all neglected.

Based on Equation (9), the performance of the one-tap normalized LMS carrier phase recovery in the coherent optical fiber communication systems using different modulation formats is shown in Figure 5. The optimization of the one-tap normalized LMS carrier phase recovery has been investigated and discussed in detail in Reference [27,37], where the step size varying from 0.01 to 1 has been applied. A smaller step size will degrade the BER floor due to the fast phase changing occurring in the long effective symbol average-span, while a larger step size will degrade the signal-to-noise ratio (SNR) sensitivity in the one-tap normalized LMS carrier phase recovery. The optimal step sizes for different effective linewidths have been studied, with a resolution of 0.005 used in the optimization process [37]. In this section we assume that all the operations of the one-tap normalized LMS algorithm have been optimized. It can be found in Figure 5 that the one-tap normalized LMS carrier phase recovery algorithm is very sensitive to the phase noise variance and the modulation formats, especially when phase noise variance is less than 0.1 .

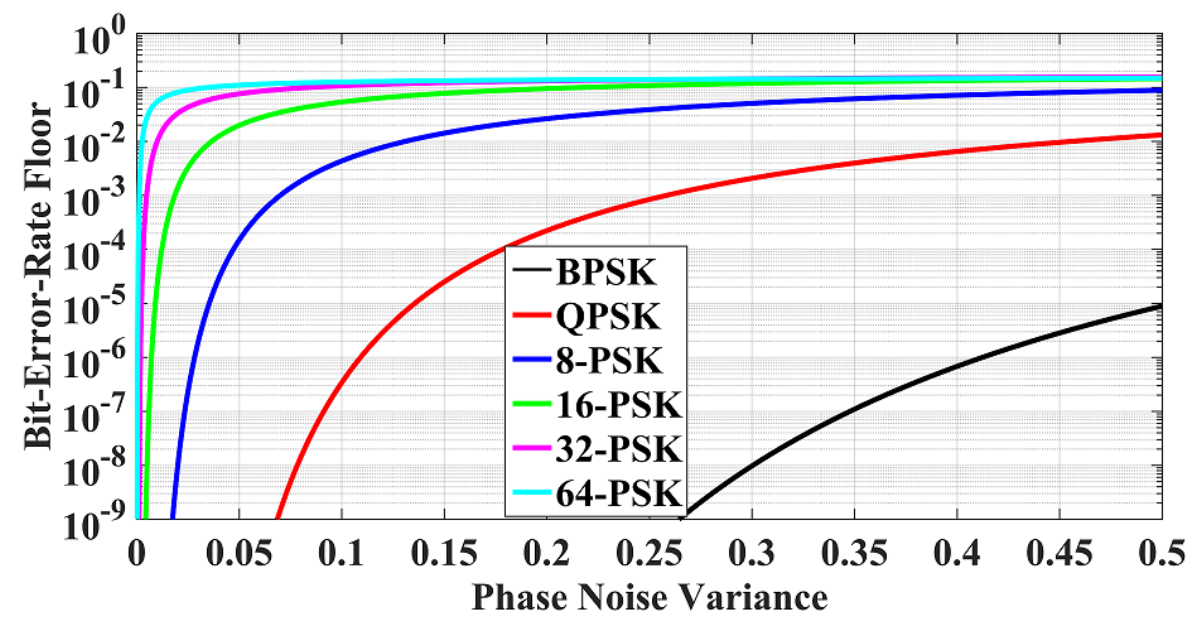

Figure 5. Bit-error-rate (BER) floors versus phase noise variance in the one-tap normalized LMS carrier phase recovery in the coherent optical transmission systems using different modulation formats.

In the case of back-to-back $(\mathrm{BtB})$ or without considering EEPN, the performance of BER floors versus laser linewidths in the one-tap normalized LMS carrier phase estimation has been studied in Figure 6 based on the analysis in Equations (1) and (9). The indicated linewidth value in Figure 6 is the 3-dB linewidth for both the transmitter laser and the LO laser. It can be seen that the BER floors of the one-tap normalized LMS carrier phase recovery are deteriorated with the increment of the modulation format levels and the laser linewidths. The degradations due to the laser phase noise (laser linewidths) are more drastic for higher-level modulation formats.

Considering the impact of EEPN, the effective phase noise variance will increase with the increment of the transmission distance and the laser linewidth. As shown in Figure 7, the BER floors 
of the one-tap normalized LMS carrier phase recovery has been investigated for different transmission distances, when the linewidths of both the Tx and the LO lasers are set to $1 \mathrm{MHz}$. It can be found that the performance of the one-tap normalized LMS carrier phase recovery is degraded significantly with the increment of transmission distances, and this effect is more severe for higher-level modulation formats due to less tolerance to laser phase noise and EEPN.

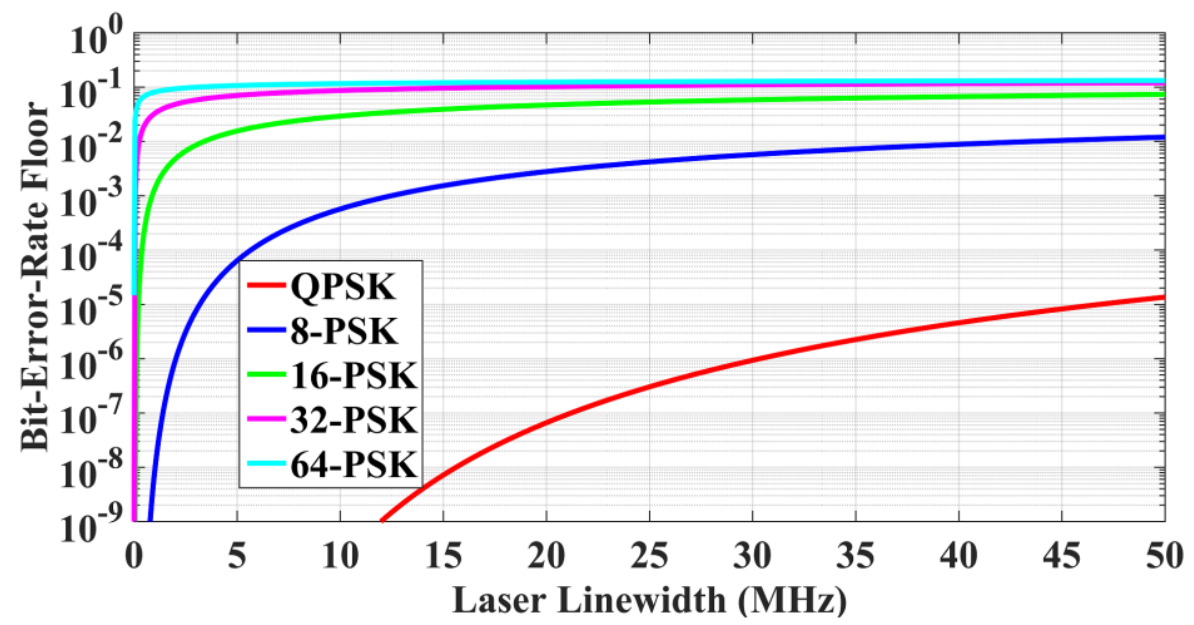

Figure 6. BER floors versus laser linewidths in the one-tap normalized LMS carrier phase recovery in the optical fiber transmission systems using different modulation formats. The indicated linewidth value is the 3-dB linewidth for both the Tx and the LO lasers.

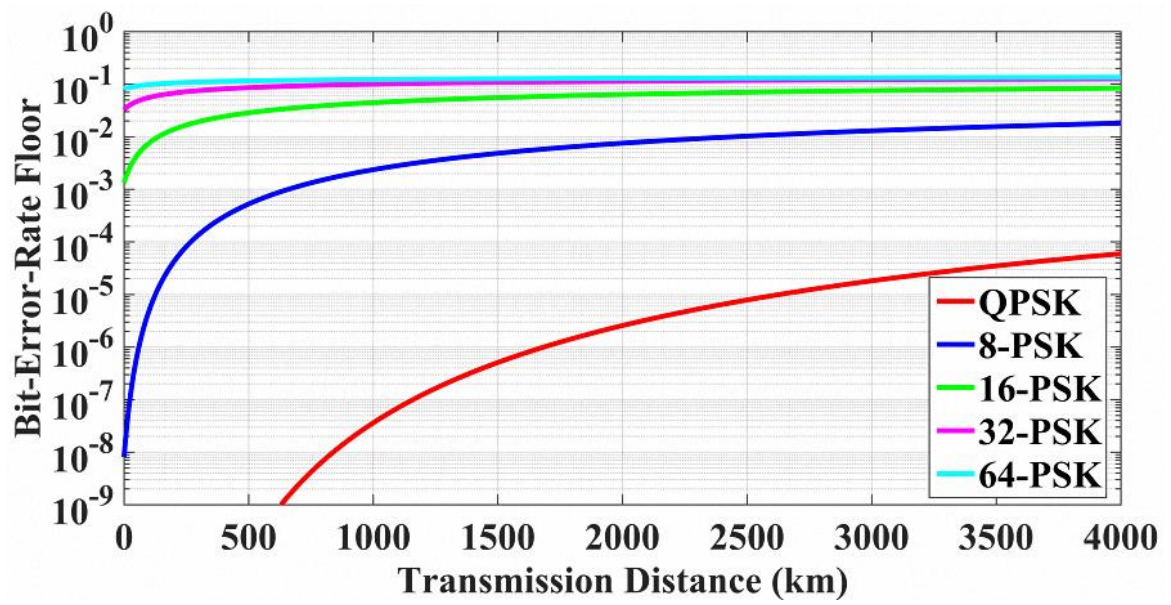

Figure 7. BER floors versus transmission distances in the one-tap normalized LMS carrier phase recovery in the coherent optical transmission systems using different modulation formats, considering the equalization enhanced phase noise. Both the Tx and LO lasers linewidths are $1 \mathrm{MHz}$.

The performance of the block-wise average carrier phase recovery approach has also been investigated, as shown in Figures 8-10. The BER floors versus different phase noise variances (or effective phase noise variance) in the block-wise average carrier phase recovery is described in Figure 8. In Figure 8a, the performance of the block-wise average algorithm is studied in terms of different block lengths in the 8-PSK optical transmission system. It can be found that the phase noise (or effective phase noise) induced BER floor in the block-wise average carrier phase recovery algorithm is increased with the increment of block length. Generally, a smaller block length will lead to a lower phase noise induced BER floor due to a more accurate estimation of carrier phase, while a larger block length is more effective for mitigating the amplitude noise (such as the amplified spontaneous emission-ASE—noise) to improve the SNR sensitivity. In practical transmission systems, the optimal 
block length is determined by considering the trade-off between the phase noise and the amplitude noise. As an example, the block length of $N_{B W A}=11$ is employed in all subsequent analyses, if the value is not specified. Based on Equations (12) and (13), the performance of the block-wise average carrier phase recovery in the coherent optical communication systems using different modulation formats is shown in Figure $8 b$, where the block length is 11 . It can be found in Figure $8 b$ that the block-wise average carrier phase recovery algorithm is also very sensitive to the phase noise variance and the modulation formats, when phase noise variance is less than 0.1 .

Based on the analyses in Equations (1), (12) and (13), the BER floors versus laser linewidths in the block-wise average carrier phase recovery for the back-to-back case or without considering EEPN has been studied in Figure 9, where the indicated linewidth value is the 3-dB linewidth for both the transmitter laser and the LO laser. It can be found that the BER floors in the block-wise average carrier phase recovery are also degraded with the increment of modulation format levels and laser linewidths. The degradations due to the laser phase noise (laser linewidths) are also more severe for higher-level modulation formats.

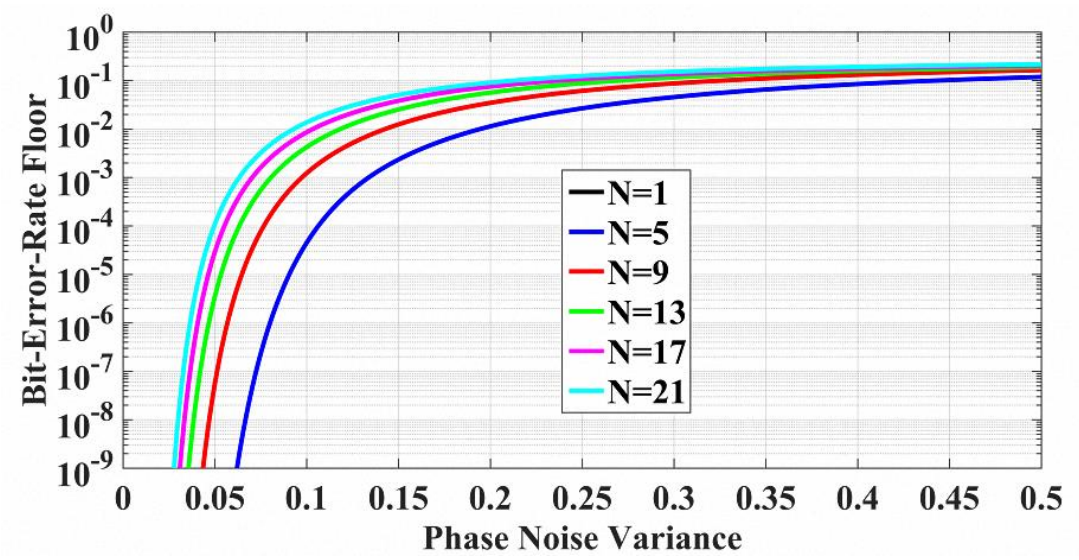

(a)

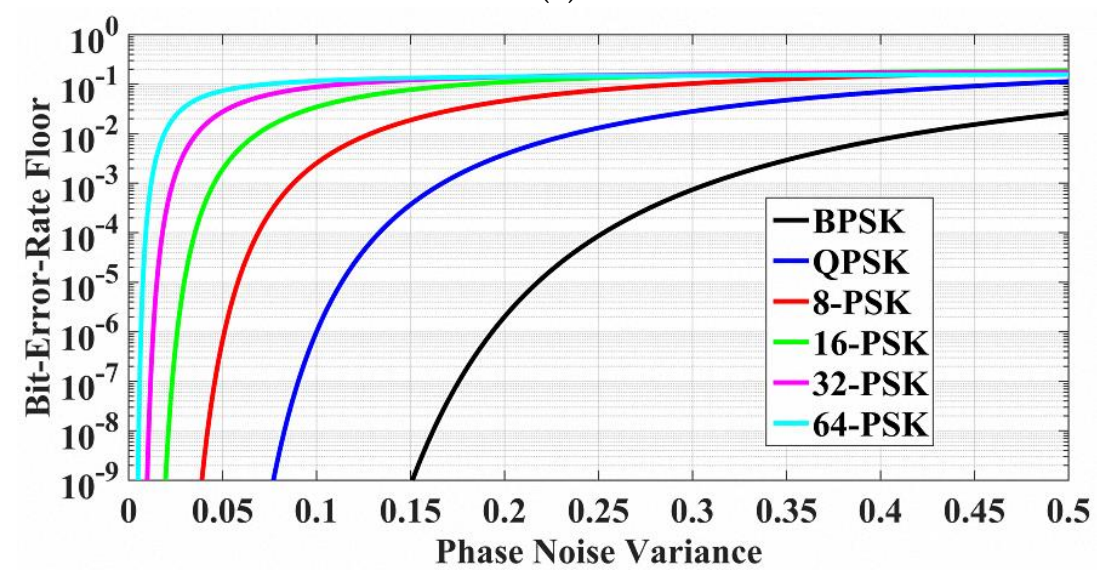

(b)

Figure 8. BER floors versus phase noise variances in the block-wise average carrier phase recovery in the coherent optical transmission systems. (a) Different block lengths in the 8-PSK transmission system; (b) different modulation formats with the block length of 11 . 


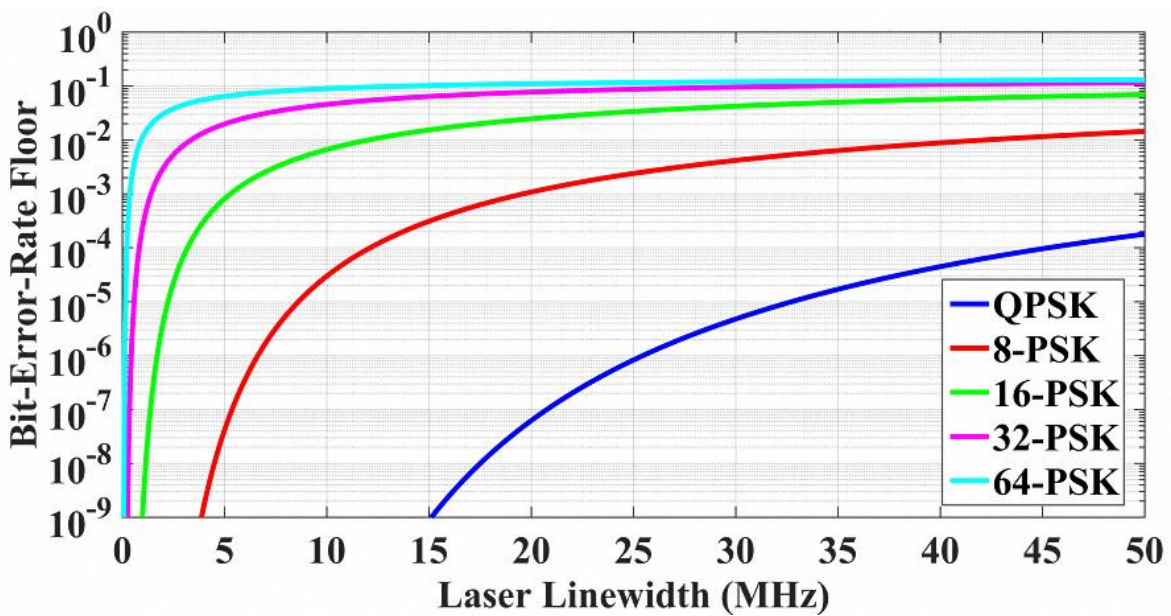

Figure 9. BER floors versus laser linewidths in the block-wise average carrier phase recovery in the coherent optical transmission systems using different modulation formats. The block length is 11, and the indicated linewidth value is the 3-dB linewidth for both the Tx and the LO lasers.

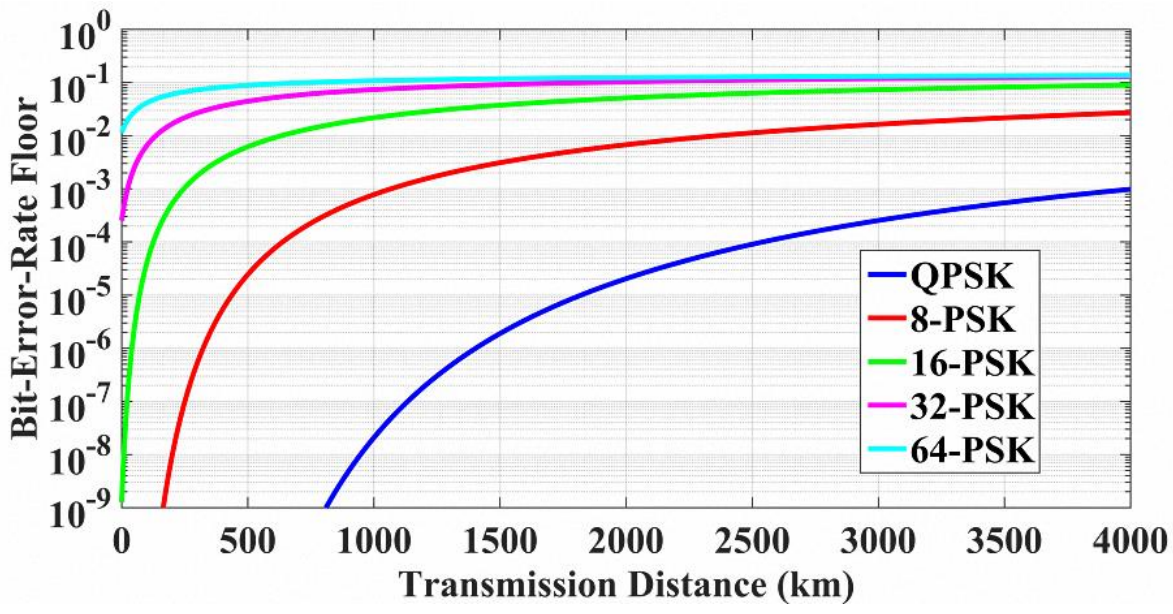

Figure 10. BER floors versus transmission distances in the block-wise average carrier phase recovery in the coherent optical transmission systems using different modulation formats. The block length is 11, and the linewidth of both the Tx and the LO lasers are $1 \mathrm{MHz}$.

As shown in Figure 10, the BER floors of the block-wise average carrier phase recovery have been investigated for different transmission distances by considering the impact of EEPN, where the linewidths of both the Tx and the $\mathrm{LO}$ lasers are set to $1 \mathrm{MHz}$. It can be found that the performance of the block-wise average carrier phase recovery is degraded significantly with the increment of transmission distances, and this effect is more severe for higher-level modulation formats, since the EEPN influence will scale with the increment of transmission distances and modulation formats.

From Figures 11-13, the performance of the Viterbi-Viterbi carrier phase recovery approach has been investigated in terms of the phase noise variances, the laser linewidths and the transmission distances. The BER floors versus the phase noise variances in the Viterbi-Viterbi carrier phase recovery algorithm is studied in Figure 11. In Figure 11a, the BER floors are also studied in terms of different block lengths in the Viterbi-Viterbi CPR algorithm for the 8-PSK optical transmission system. Similar to the block-wise average algorithm, a smaller block length in the Viterbi-Viterbi carrier phase recovery will generate a lower phase noise induced BER floor; in contrast, a larger block length is more tolerant to the amplitude noise and will lead to better SNR sensitivity. The optimal block length is again determined by considering the trade-off between phase noise and amplitude noise. It can be found 
that the phase noise induced BER floors in the Viterbi-Viterbi carrier phase recovery algorithm are also deteriorated with the increment of block lengths. Similar to the block-wise average algorithm, the block length of $N_{V V}=11$ is also selected as an example in the Viterbi-Viterbi carrier phase recovery to consider the mitigation of both the phase noise and the amplitude noise in practical applications. Based on Equation (15), the performance of the Viterbi-Viterbi carrier phase recovery in the coherent optical communication systems using different modulation formats is shown in Figure 11b, where the block length is 11. It is found in Figure 11b that the Viterbi-Viterbi carrier phase recovery algorithm is also very sensitive to the phase noise variance and the modulation formats, when phase noise variance is less than 0.15 .

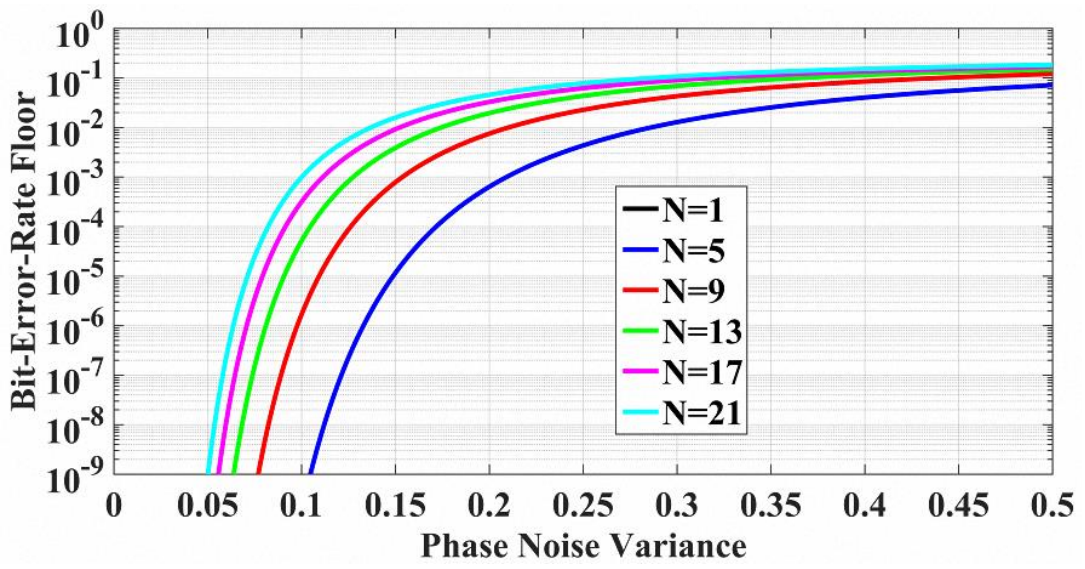

(a)

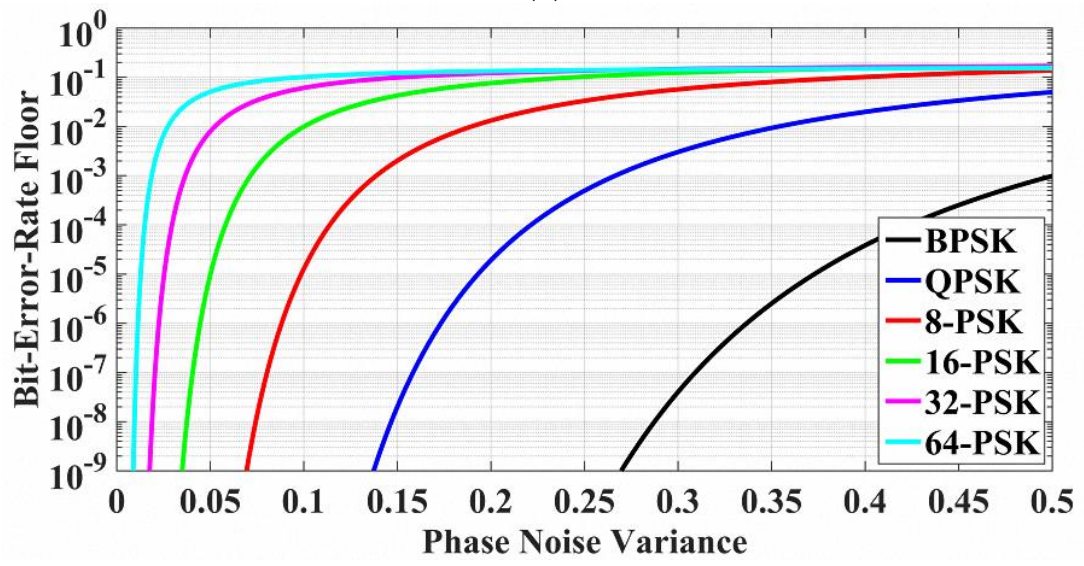

(b)

Figure 11. BER floors versus phase noise variances in the Viterbi-Viterbi carrier phase recovery in the coherent optical transmission systems. (a) Different block lengths in the 8-PSK transmission system; (b) different modulation formats with the block length of 11 .

Without considering EEPN (or for the back-to-back case), the BER floors versus laser linewidths in the Viterbi-Viterbi carrier phase recovery have been studied in Figure 12 based on the analyses in Equations (1) and (15), where the indicated linewidth value is again the 3-dB linewidth for both the transmitter laser and the LO laser. It can be found that the BER floors are also degraded significantly with the increment of laser linewidths, and this degradation is also more severe with the increment of modulation format levels.

As shown in Figure 13, the BER floors of the Viterbi-Viterbi carrier phase recovery have also been investigated for different transmission distances considering the impact of EEPN, where the linewidths of the Tx and the LO lasers are both set to $1 \mathrm{MHz}$. It can be found that in the Viterbi-Viterbi carrier phase recovery, the EEPN influence will also increase with the increment of transmission 
distances and modulation formats. The performance of the Viterbi-Viterbi algorithm is degraded significantly with the increment of transmission distances, and this effect is more serious for higher-level modulation formats.

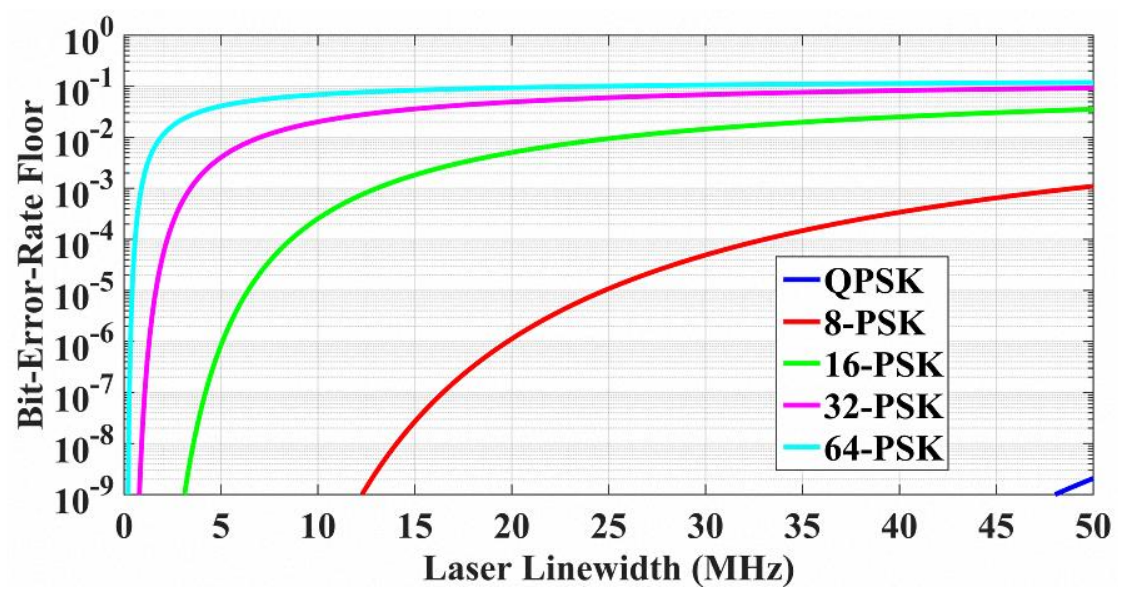

Figure 12. BER floors versus laser linewidths in the Viterbi-Viterbi carrier phase recovery in the coherent optical transmission systems using different modulation formats. The block length is 11, and the indicated linewidth value is the 3-dB linewidth for both the Tx and the LO lasers.

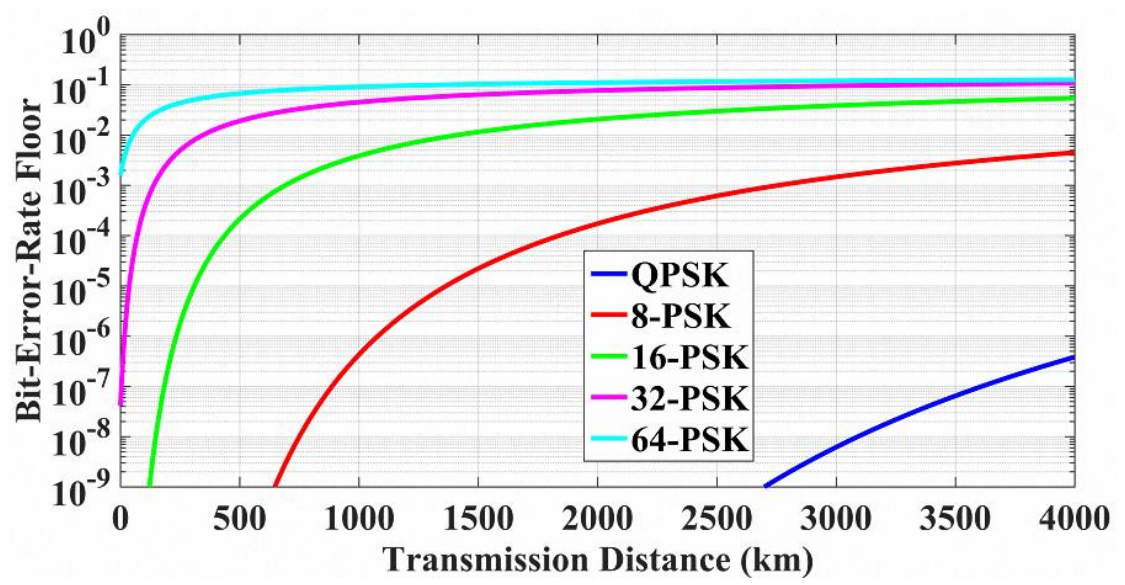

Figure 13. BER floors versus transmission distances in the Viterbi-Viterbi carrier phase recovery in the coherent optical transmission systems using different modulation formats. The block length is 11 , and the linewidth of both the Tx and the LO lasers are $1 \mathrm{MHz}$.

The comparisons of the one-tap normalized LMS, the block-wise average, and the Viterbi-Viterbi carrier phase recovery algorithms have also been investigated in detail. The BER floors versus different phase noise variances in the above three carrier phase recovery algorithms in the 8-PSK optical fiber communication system have been studied and are shown in Figure 14, where the block length in the block-wise average and the Viterbi-Viterbi algorithms varies from 5 to 17, in Figure 14a-c, respectively. It can be seen that the phase noise induced BER floors in the block-wise average and Viterbi-Viterbi algorithms are degraded with increment of the block length, and the one-tap normalized LMS algorithm keeps the same performance due to its optimized operation. It is also found in Figure 14 that for the small phase noise variance (or effective phase noise variance), the Viterbi-Viterbi carrier phase recovery algorithm outperforms the one-tap normalized LMS and the block-wise average algorithms, while for the large phase noise variance (or effective phase noise variance), the one-tap normalized LMS algorithm shows a better performance than the other two algorithms in the carrier phase recovery. 


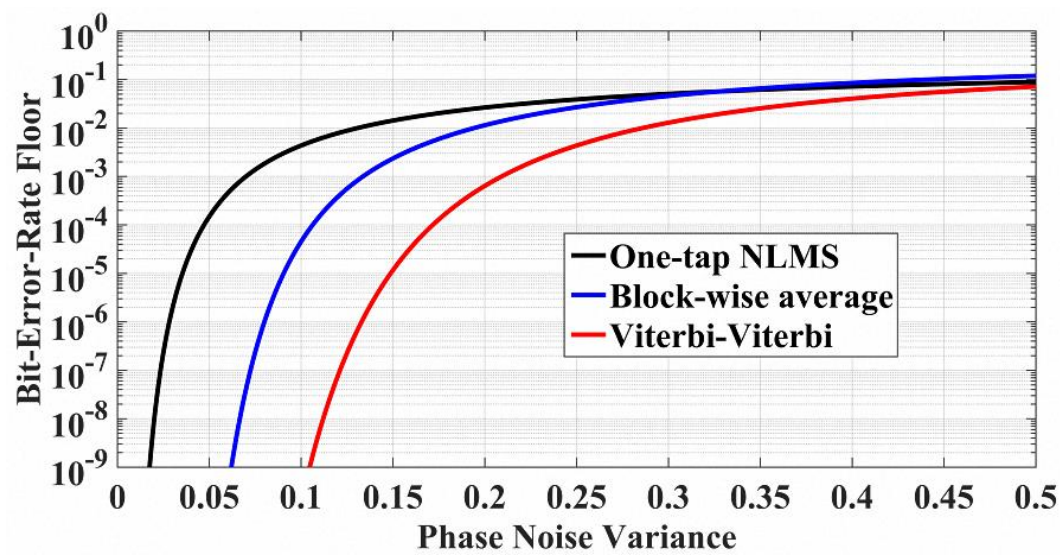

(a)

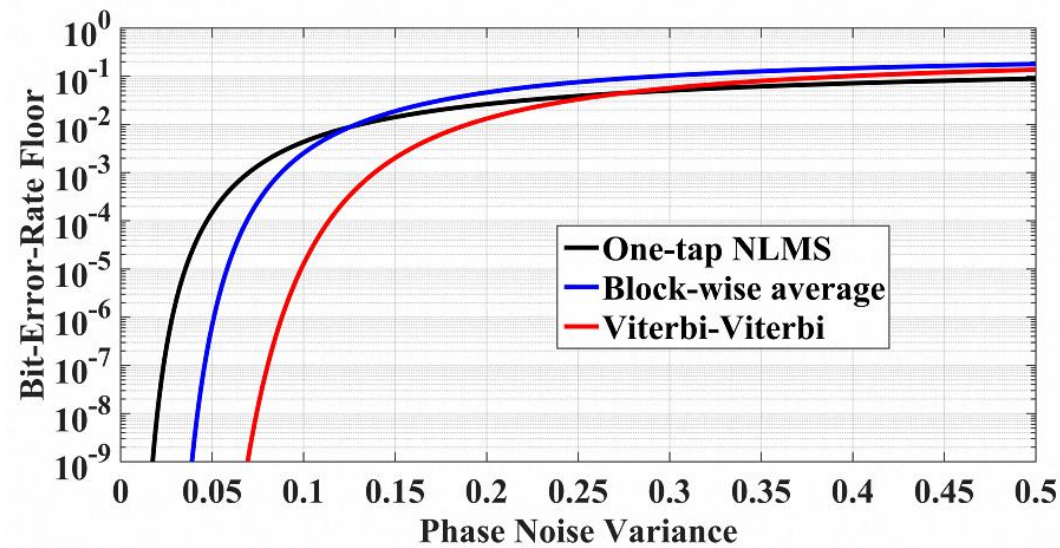

(b)

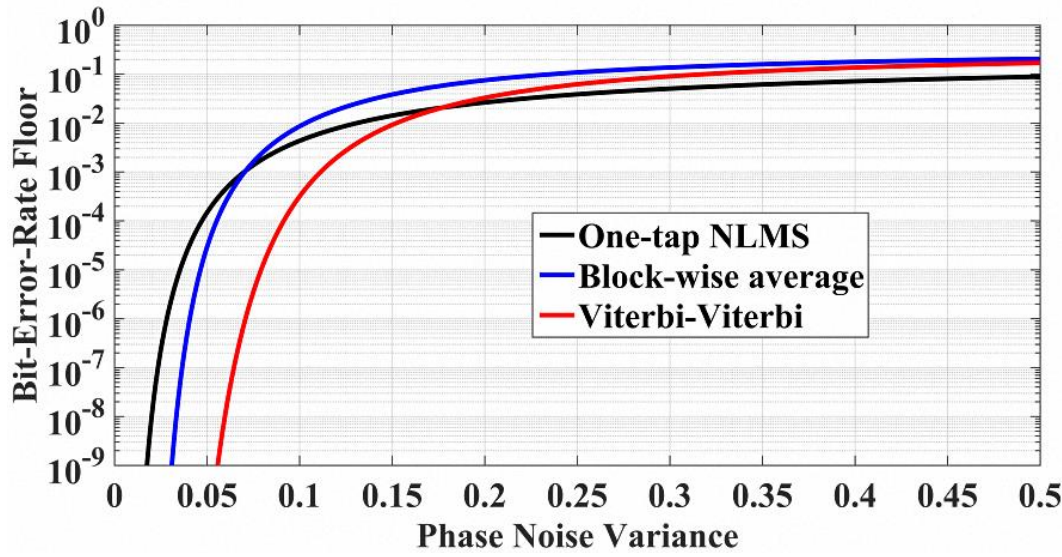

(c)

Figure 14. BER floors versus different phase noise variances in the three carrier phase recovery algorithms in the 8-PSK optical fiber communication systems. (a) Block length of the BWA and VV algorithms is 5; (b) block length of the BWA and VV algorithms is 11; (c) block length of the BWA and VV algorithms is 17 .

As shown in Figures 15 and 16, the comparison of the one-tap normalized LMS, the block-wise average, and the Viterbi-Viterbi carrier phase recovery algorithms has also been investigated in terms of different modulation formats. Here a block length of 11 is used in the block-wise average and the Viterbi-Viterbi algorithms. It can be found in Figure 15 that the BER floors in all the three algorithms are increased with the increment of modulation formats, but the variation in the one-tap normalized LMS CPR algorithm is larger than in the other two algorithms. Therefore, the one-tap normalized LMS algorithm is more sensitive to the level of the modulation formats. 


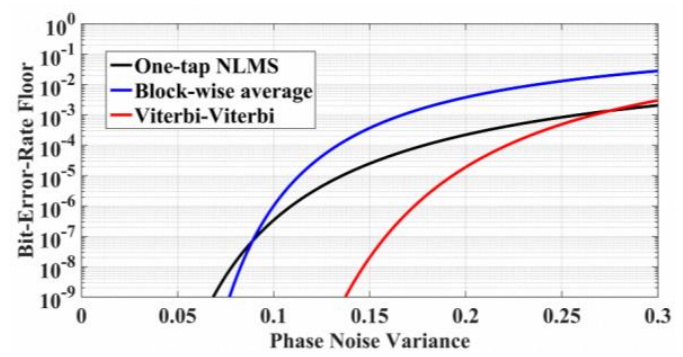

(a)

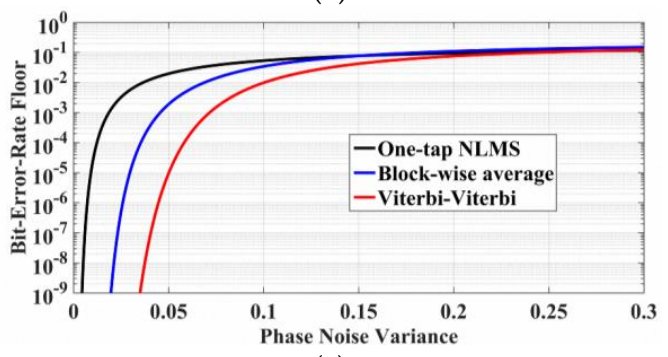

(c)

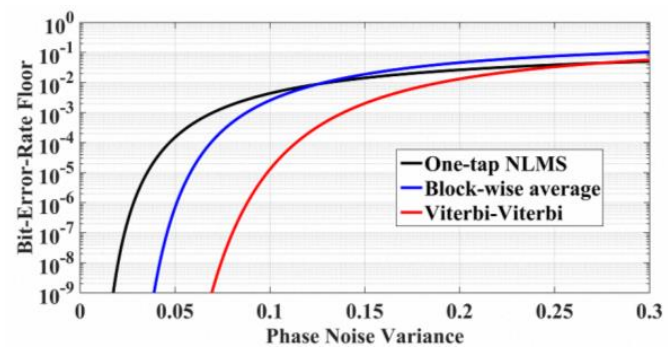

(b)

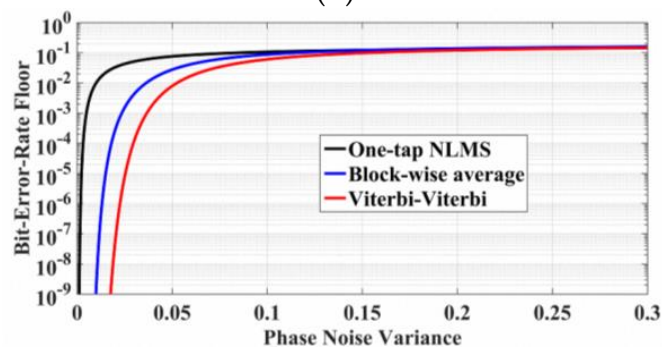

(d)

Figure 15. BER floors versus different phase noise variances in the three carrier phase recovery algorithms in the optical fiber communication systems using different modulation formats. Block lengths of the BWA and VV algorithms are both 11. (a) QPSK system; (b) 8-PSK system; (c) 16-PSK system; (d) 32-PSK system.

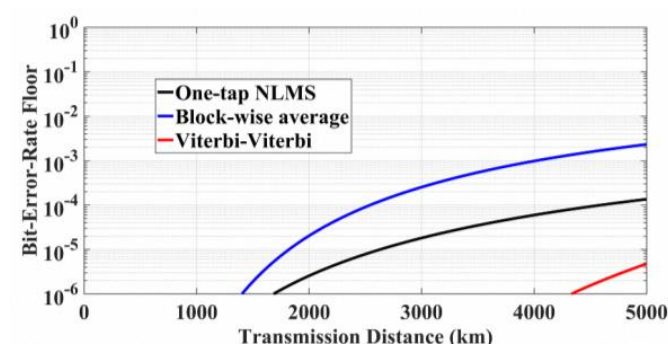

(a)

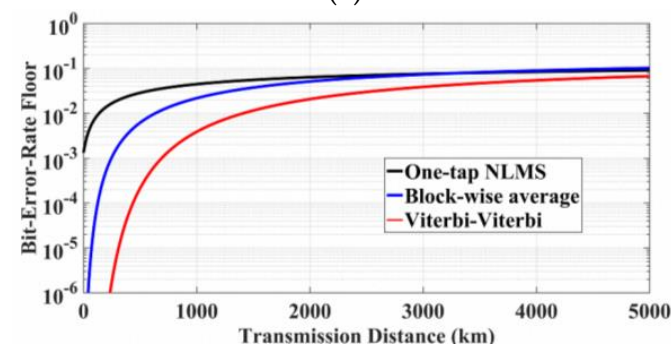

(c)

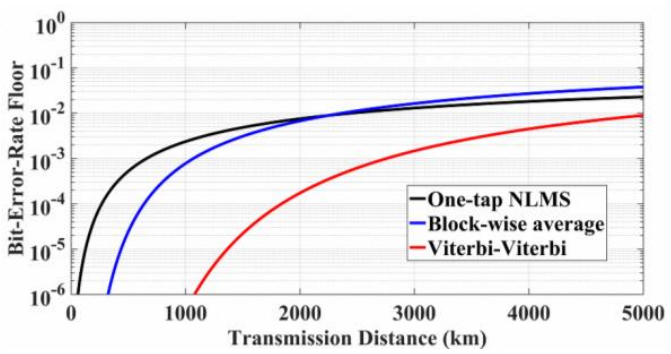

(b)

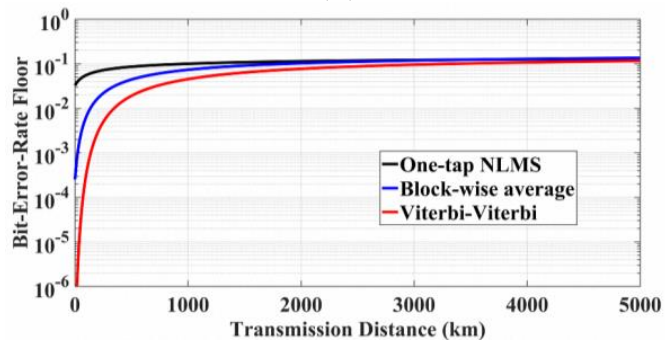

(d)

Figure 16. BER floors versus different transmission distances in the three carrier phase recovery algorithms in the optical fiber communication systems using different modulation formats. Linewidths of the transmitter and LO lasers are both $1 \mathrm{MHz}$, and block lengths of the BWA and VV CPR algorithms are both 11. (a) QPSK system; (b) 8-PSK system; (c) 16-PSK system; (d) 32-PSK system.

Figure 16 shows the performance of BER floors versus transmission distances in the three carrier phase recovery methods under different modulation formats, where the linewidths of both the transmitter and the LO lasers are set as $1 \mathrm{MHz}$ and the transmission distance are set from $0 \mathrm{~km}$ to $5000 \mathrm{~km}$. It can also be found in Figure 16 that, similar to Figure 15, the one-tap normalized LMS algorithm is more sensitive to the level of the modulation formats than the block-wise average and the Viterbi-Viterbi CPR algorithms. In addition, the Viterbi-Viterbi algorithm outperforms the other two carrier phase recovery approaches when the transmission distance varies from $0 \mathrm{~km}$ to $5000 \mathrm{~km}$, 
while the differences between the three carrier phase recovery algorithms become smaller with the increment of modulation formats.

\subsection{Ideal Spectral Efficiency in Carrier Phase Recovery}

For the binary symmetric channel (binary input and output alphabets, symmetric transition probability), the coding rate $R_{C}$ in the $n$-PSK optical fiber communication systems (assuming an ideal hard-decision forward error correction coding) can be expressed as follows [53],

$$
R_{C}=1+B E R \cdot \log _{2}(B E R)+(1-B E R) \cdot \log _{2}(1-B E R) .
$$

Correspondingly, the ideal spectral efficiency (assuming an ideal hard-decision forward error correction coding) in the carrier phase recovery in the $n$-PSK coherent optical fiber communication systems can be calculated as:

$$
S E=R_{C} \cdot N_{p} \cdot \log _{2}(n),
$$

where $S E$ is the ideal spectral efficiency, $n$ is the modulation format level of the communication system, $N_{p}$ is the number of polarization states. The BER limits in Equation (16) can be obtained from the BER floors in the three carrier phase recovery approaches according to Equations (9), (12) and (15), respectively.

\subsection{Complexity of Carrier Phase Recovery Approaches}

The computational complexity is always a significant consideration and criterion for the DSP algorithms. Here the complexity of the one-tap normalized LMS, the block-wise average, and the Viterbi-Viterbi carrier phase recovery algorithms has been investigated in terms of the number of the complex multiplications per recovered symbol, which are shown in Table 1 ( $n$ is the level of modulation formats). It is found that the computational complexity of the one-tap normalized LMS algorithm is independent from the modulation formats, while the complexity of the block-wise average and the Viterbi-Viterbi CPR algorithms scales linearly with the level of modulation formats. Note that the computations in the pre-convergence of the one-tap normalized LMS algorithm also has to be considered in the practical applications.

Table 1. Complexity of carrier phase recovery approaches (complex multiplications per symbol).

\begin{tabular}{ccc}
\hline One-Tap Normalized LMS & Block-Wise Average & Viterbi-Viterbi \\
\hline 5 & $n$ & $n$ \\
\hline
\end{tabular}

All the above analyses are based on the carrier phase recovery in the $n$-PSK coherent optical communication systems; however, all these discussions can be directly extended into the circular $n$-QAM transmission systems. Meanwhile, although the $n$-PSK signals have a lower tolerance to the ASE noise than the multi-amplitude signals (such as $n$-QAM signals), the $n$-PSK signals will have a better tolerance to fiber nonlinearities due to the constant amplitudes $[54,55]$.

\section{Conclusions}

Theoretical analyses of the carrier phase recovery in long-haul high-speed $n$-PSK ( $n$-level phase shift keying) coherent optical fiber communication systems, using the one-tap normalized least-mean-square (LMS), block-wise average, and Viterbi-Viterbi algorithms, have been investigated and described in detail, considering both the laser phase noise and the equalization enhanced phase noise. The expressions for the estimated carrier phase in these three algorithms have been presented, and the bit-error-rate (BER) performance such as the BER floors, has been predicted analytically. Comparative studies of the one-tap normalized LMS, block-wise average, and Viterbi-Viterbi algorithms have also been carried out. It has been found that the Viterbi-Viterbi carrier phase recovery 
algorithm outperforms the one-tap normalized LMS and the block-wise average algorithms for small phase noise variance (or effective phase noise variance), while the one-tap normalized LMS algorithm shows a better performance than the other two algorithms in the carrier phase recovery for large phase noise variance (or effective phase noise variance). In addition, the one-tap normalized LMS carrier phase recovery algorithm is more sensitive to the level of modulation formats than the other two algorithms.

The BER floors in this paper were discussed and analyzed based on the influence from laser phase noise and equalization enhanced phase noise in the long-haul $n$-PSK transmission systems, and this represents the system limits from laser phase noise and equalization enhanced phase noise. In addition, signal degradation from fiber nonlinearities is also a significant effect in such communication systems. Therefore, the actual BER floors will be determined by involving the impacts from amplified spontaneous emission (ASE) noise, laser phase noise, equalization enhanced phase noise and fiber nonlinearities, which will be investigated in our future work.

Acknowledgments: This work is supported in part by UK Engineering and Physical Sciences Research Council (project UNLOC EP/J017582/1), in part by European Commission Research Council FP7-PEOPLE-2012-IAPP (project GRIFFON, No. 324391), in part by European Commission Research Council FP7-PEOPLE-2013-ITN (project ICONE, No. 608099), and in part by Swedish Research Council Vetenskapsradet (No. 0379801).

Author Contributions: T.X. presented the basic idea and carried out the analytical calculations and discussions. G.J., S.P., J.L., T.L., Y.Z. and P.B. contributed to developing the research ideas and were involved in the discussion of results. T.X. wrote the main manuscript and prepared the figures. All authors reviewed the manuscript and gave the final approval for publication.

Conflicts of Interest: The authors declare no conflict of interest.

\section{References}

1. Essiambre, R.J.; Foschini, G.J.; Kramer, G.; Winzer, P.J. Capacity limits of information transport in fiber-optic networks. Phys. Rev. Lett. 2008, 101, 163901. [CrossRef] [PubMed]

2. Bayvel, P.; Maher, R.; Xu, T.; Liga, G.; Shevchenko, N.A.; Lavery, D.; Alvarado, A.; Killey, R.I. Maximising the optical network capacity. Philos. Trans. R. Soc. A 2016, 374, 20140440. [CrossRef] [PubMed]

3. Essiambre, R.J.; Tkach, R.W. Capacity trends and limits of optical communication networks. Proc. IEEE 2012, 100, 1035-1055. [CrossRef]

4. Kaminow, I.; Li, T.; Willner, A.E. Optical Fiber Telecommunications VB: System and Networks; Academic Press: Oxford, UK, 2010.

5. Agrawal, G.P. Fiber-Optic Communication Systems, 4th ed.; John Wiley \& Sons, Inc.: New York, NY, USA, 2010.

6. Li, Y.; Xu, T.; Jia, D.; Jing, W.; Hu, H.; Yu, J.; Zhang, Y. Dynamic dispersion compensation in a 40 Gb/s single-channeled optical fiber communication system. Acta Opt. Sin. 2007, 27, 1161-1165.

7. Xu, T.; Li, J.; Jacobsen, G.; Popov, S.; Djupsjöbacka, A.; Schatz, R.; Zhang, Y.; Bayvel, P. Field trial over $820 \mathrm{~km}$ installed SSMF and its potential Terabit/s superchannel application with up to 57.5-Gbaud DP-QPSK transmission. Opt. Commun. 2015, 353, 133-138. [CrossRef]

8. Galili, M.; Hu, H.; Mulvad, H.C.H.; Medhin, A.K.; Clausen, A.; Oxenløwe, L.K. Optical systems for ultra-high-speed TDM networking. Photonics 2014, 1, 83-94. [CrossRef]

9. Ip, E.; Lau, A.P.T.; Barros, D.J.F.; Kahn, J.M. Coherent detection in optical fiber systems. Opt. Express 2008, 16, 753-791. [CrossRef] [PubMed]

10. Xu, T.; Jacobsen, G.; Popov, S.; Li, J.; Vanin, E.; Wang, K.; Friberg, A.T.; Zhang, Y. Chromatic dispersion compensation in coherent transmission system using digital filters. Opt. Express 2010, 18, 16243-16257. [CrossRef] [PubMed]

11. Taylor, M.G. Coherent detection method using DSP for demodulation of signal and subsequent equalization of propagation impairments. IEEE Photonics Technol. Lett. 2004, 16, 674-676. [CrossRef]

12. Savory, S.J. Digital filters for coherent optical receivers. Opt. Express 2008, 16, 804-817. [CrossRef] [PubMed]

13. Xu, T.; Jacobsen, G.; Popov, S.; Li, J.; Wang, K.; Friberg, A.T. Normalized LMS digital filter for chromatic dispersion equalization in 112-Gbit/s PDM-QPSK coherent optical transmission system. Opt. Commun. 2010, 283, 963-967. [CrossRef] 
14. Kudo, R.; Kobayashi, T.; Ishihara, K.; Takatori, Y.; Sano, A.; Miyamoto, Y. Coherent optical single carrier transmission using overlap frequency domain equalization for long-haul optical systems. J. Lightwave Technol. 2009, 27, 3721-3728. [CrossRef]

15. Ip, E.; Kahn, J.M. Digital equalization of chromatic dispersion and polarization mode dispersion. J. Lightwave Technol. 2007, 25, 2033-2043. [CrossRef]

16. Xu, T.; Jacobsen, G.; Popov, S.; Li, J.; Friberg, A.T.; Zhang, Y. Carrier phase estimation methods in coherent transmission systems influenced by equalization enhanced phase noise. Opt. Commun. 2013, 293, 54-60. [CrossRef]

17. Liga, G.; Xu, T.; Alvarado, A.; Killey, R.; Bayvel, P. On the performance of multichannel digital backpropagation in high-capacity long-haul optical transmission. Opt. Express 2014, 22, 30053-30062. [CrossRef] [PubMed]

18. Maher, R.; Xu, T.; Galdino, L.; Sato, M.; Alvarado, A.; Shi, K.; Savory, S.J.; Thomsen, B.C.; Killey, R.I.; Bayvel, P. Spectrally shaped DP-16QAM super-channel transmission with multi-channel digital back propagation. Sci. Rep. 2015, 5, 08214. [CrossRef] [PubMed]

19. Shannon, C.E. A mathematical theory of communication. Bell Syst. Tech. J. 1948, 27, 379-423. [CrossRef]

20. Essiambre, R.J.; Kramer, G.; Winzer, P.J.; Foschini, G.J.; Goebel, B. Capacity limits of optical fiber networks. J. Lightwave Technol. 2010, 28, 662-701. [CrossRef]

21. Kazovsky, L.G. Impact of laser phase noise on optical heterodyne communication systems. J. Opt. Commun. 1986, 7, 66-78. [CrossRef]

22. Zhang, S.; Kam, P.Y.; Yu, C.; Chen, J. Laser linewidth tolerance of decision-aided maximum likelihood phase estimation in coherent optical M-ary PSK and QAM systems. IEEE Photonics Technol. Lett. 2009, 21, 1075-1077. [CrossRef]

23. Taylor, M.G. Phase estimation methods for optical coherent detection using digital signal processing. J. Lightwave Technol. 2009, 17, 901-914. [CrossRef]

24. Fatadin, I.; Ives, D.; Savory, S.J. Differential carrier phase recovery for QPSK optical coherent systems with integrated tunable lasers. Opt. Express 2013, 21, 10166-10171. [CrossRef] [PubMed]

25. Ip, E.; Kahn, J.M. Feedforward carrier recovery for coherent optical communications. J. Lightwave Technol. 2007, 25, 2675-2692. [CrossRef]

26. Goldfarb, G.; Li, G. BER estimation of QPSK homodyne detection with carrier phase estimation using digital signal processing. Opt. Express 2006, 14, 8043-8053. [CrossRef] [PubMed]

27. Mori, Y.; Zhang, C.; Igarashi, K.; Katoh, K.; Kikuchi, K. Unrepeated 200-km transmission of 40-Gbit/s 16-QAM signals using digital coherent receiver. Opt. Express 2009, 17, 1435-1441. [CrossRef] [PubMed]

28. Fatadin, I.; Ives, D.; Savory, S.J. Blind equalization and carrier phase recovery in a 16-QAM optical coherent system. J. Lightwave Technol. 2009, 27, 3042-3049. [CrossRef]

29. Kikuchi, K. Phase-diversity homodyne detection of multilevel optical modulation with digital carrier phase estimation. IEEE J. Sel. Top. Quant. Electron. 2006, 12, 563-570. [CrossRef]

30. Ly-Gagnon, D.S.; Tsukamoto, S.; Katoh, K.; Kikuchi, K. Coherent detection of optical quadrature phase-shift keying signals with carrier phase estimation. J. Lightwave Technol. 2006, 24, 12-21. [CrossRef]

31. Viterbi, A.J.; Viterbi, A.M. Nonlinear estimation of PSK-modulated carrier phase with application to burst digital transmission. IEEE Trans. Inf. Theory 1983, 29, 543-551. [CrossRef]

32. Jacobsen, G.; Xu, T.; Popov, S.; Sergeyev, S. Study of EEPN mitigation using modified RF pilot and Viterbi-Viterbi based phase noise compensation. Opt. Express 2013, 21, 12351-12362. [CrossRef] [PubMed]

33. Shieh, W.; Ho, K.P. Equalization-enhanced phase noise for coherent detection systems using electronic digital signal processing. Opt. Express 2008, 16, 15718-15727. [CrossRef] [PubMed]

34. Xie, C. Local oscillator phase noise induced penalties in optical coherent detection systems using electronic chromatic dispersion compensation. In Proceedings of the Conference on Optical Fiber Communication (OFC), San Diego, CA, USA, 22-26 March 2009.

35. Lau, A.P.T.; Shen, T.S.R.; Shieh, W.; Ho, K.P. Equalization-enhanced phase noise for $100 \mathrm{~Gb} / \mathrm{s}$ transmission and beyond with coherent detection. Opt. Express 2010, 18, 17239-17251. [CrossRef] [PubMed]

36. Fatadin, I.; Savory, S.J. Impact of phase to amplitude noise conversion in coherent optical systems with digital dispersion compensation. Opt. Express 2010, 18, 16273-16278. [CrossRef] [PubMed] 
37. Xu, T.; Jacobsen, G.; Popov, S.; Li, J.; Friberg, A.T.; Zhang, Y. Analytical estimation of phase noise influence in coherent transmission system with digital dispersion equalization. Opt. Express 2011, 19, 7756-7768. [CrossRef] [PubMed]

38. Ho, K.P.; Lau, A.P.T.; Shieh, W. Equalization-enhanced phase noise induced time jitter. Opt. Lett. 2011, 36, 585-587. [CrossRef] [PubMed]

39. Jacobsen, G.; Xu, T.; Popov, S.; Li, J.; Friberg, A.T.; Zhang, Y. EEPN and CD study for coherent optical nPSK and nQAM systems with RF pilot based phase noise compensation. Opt. Express 2012, 20, 8862-8870. [CrossRef] [PubMed]

40. Xu, T.; Jacobsen, G.; Popov, S.; Li, J.; Sergeyev, S.; Friberg, A.T.; Zhang, Y. Analytical BER performance in differential $n$-PSK coherent transmission system influenced by equalization enhanced phase noise. Opt. Commun. 2015, 334, 222-227. [CrossRef]

41. Xu, T.; Liga, G.; Lavery, D.; Thomson, B.C.; Savory, S.J.; Killey, R.I.; Bayvel, P. Equalization enhanced phase noise in Nyquist-spaced superchannel transmission systems using multi-channel digital back-propagation. Sci. Rep. 2015, 5, 13990. [CrossRef] [PubMed]

42. Zhuge, Q.; Morsy-Osman, M.H.; Plant, D.V. Low overhead intra-symbol carrier phase recovery for reduced-guard-interval CO-OFDM. J. Lightwave Technol. 2013, 31, 1158-1169. [CrossRef]

43. Jacobsen, G.; Lidón, M.; Xu, T.; Popov, S.; Friberg, A.T.; Zhang, Y. Influence of pre- and post-compensation of chromatic dispersion on equalization enhanced phase noise in coherent multilevel systems. J. Opt. Commun. 2011, 32, 257-261. [CrossRef]

44. Kakkar, A.; Navarro, J.R.; Schatz, R.; Pang, X.; Ozolins, O.; Louchet, H.; Jacobsen, G.; Popov, S. Equalization enhanced phase noise in coherent optical systems with digital pre- and post-processing. Photonics 2016, 3, 12. [CrossRef]

45. Ho, K.P.; Shieh, W. Equalization-enhanced phase noise in mode-division multiplexed systems. J. Lightwave Technol. 2013, 31, 2237-2243.

46. Shieh, W. Interaction of laser phase noise with differential-mode-delay in few-mode fiber based MIMO systems. In Proceedings of the Conference on Optical Fiber Communication (OFC), Los Angeles, CA, USA, 4-8 March 2012.

47. Colavolpe, G.; Foggi, T.; Forestieri, E.; Secondini, M. Impact of phase noise and compensation techniques in coherent optical systems. J. Lightwave Technol. 2011, 29, 2790-2800. [CrossRef]

48. Farhoudi, R.; Ghazisaeidi, A.; Rusch, L.A. Performance of carrier phase recovery for electronically dispersion compensated coherent system. Opt. Express 2012, 20, 26568-26582. [CrossRef] [PubMed]

49. Jacobsen, G.; Xu, T.; Popov, S.; Li, J.; Friberg, A.T.; Zhang, Y. Receiver implemented RF pilot tone phase noise mitigation in coherent optical nPSK and nQAM systems. Opt. Express 2011, 19, 14487-14494. [CrossRef] [PubMed]

50. Yoshida, T.; Sugihara, T.; Uto, K. DSP-based optical modulation technique for long-haul transmission. In Proceedings of the Next-Generation Optical Communication: Components, Sub-Systems, and Systems IV, San Francisco, CA, USA, 7 February 2015.

51. Vanin, E.; Jacobsen, G. Analytical estimation of laser phase noise induced BER floor in coherent receiver with digital signal processing. Opt. Express 2010, 18, 4246-4259. [CrossRef] [PubMed]

52. Kakkar, A.; Navarro, J.R.; Schatz, R.; Louchet, H.; Pang, X.; Ozolins, O.; Jacobsen, G.; Popov, S. Comprehensive study of equalization-enhanced phase noise in coherent optical systems. J. Lightwave Technol. 2015, 33, 4834-4841. [CrossRef]

53. Cover, T.M.; Thomas, J.A. Elements of Information Theory, 2nd ed.; John Wiley \& Sons, Inc.: Hoboken, NJ, USA, 2006.

54. Kojima, K.; Koike-Akino, T.; Millar, D.S.; Parsons, K. BICM capacity analysis of 8QAM-alternative modulation formats in nonlinear fiber transmission. In Proceedings of the IEEE Tyrrhenian International Workshop on Digital Communications, Florence, Italy, 22 September 2015; pp. 57-59.

55. Kojima, K.; Koike-Akino, T.; Millar, D.S.; Pajovic, M.; Parsons, K.; Yoshida, T. Investigation of low code rate DP-8PSK as an alternative to DP-QPSK. In Proceedings of the Conference on Optical Fiber Communication (OFC), Anaheim, CA, USA, 20-22 March 2016.

(C) 2016 by the authors; licensee MDPI, Basel, Switzerland. This article is an open access article distributed under the terms and conditions of the Creative Commons Attribution (CC-BY) license (http://creativecommons.org/licenses/by/4.0/). 